$17 \mid 1998$

Geistige Eigentumsrechte, was steht für die Entwicklungsländer auf dem spiel?

\title{
4. Umwelt und Entwicklung
}

\section{CpenEdition}

\section{Journals}

Electronic version

URL: http://journals.openedition.org/sjep/698

DOI: $10.4000 /$ sjep.698

ISSN: 1663-9677

Publisher

Institut de hautes études internationales et du développement

\section{Printed version}

Date of publication: 1 avril 1998

Number of pages: 191-212

ISSN: 1660-5926

\section{Electronic reference}

« 4. Umwelt und Entwicklung », Schweizerisches Jahrbuch für Entwicklungspolitik [Online], 17| 1998,

Online erschienen am: 03 August 2012, abgerufen am 08 September 2020. URL : http://

journals.openedition.org/sjep/698; DOI : https://doi.org/10.4000/sjep.698 


\section{UMWELT UND ENTWICKLUNG}

D ie Konferenz der Vereinten Nationen für Umwelt und Entwicklung (UNCED), die 1992 in Rio stattfand, markierte eine wichtige Etappe auf dem Weg hin zur nachhaltigen Entwicklung. Entscheidend ist die Sensibilisierung der Staats- und Regierungschefs der ganzen Welt, der Wirtschaftsführer und der Vertreter der verschiedenen gesellschaftlichen Gruppen für die Notwendigkeit eines neuen Denkens und Handelns. Das Konzept der nachhaltigen Entwicklung kann gesellschaftliche Solidarität, ökologische Verantwortung und wirtschaftliche Effizienz (wieder) vereinbaren. Seit dem Gipfel von Rio hat es auf internationaler, nationaler und lokaler Ebene verschiedene Anstrengungen und Initiativen zur Konkretisierung der nachhaltigen Entwicklung gegeben.

In der Schweiz hat der Bund zwei Dokumente zur Umsetzung der nachhaltigen Entwicklung im nationalen Rahmen vorbereitet und der Sondersitzung der Vereinten Nationen (auch als UNGASS oder "Rio +5 » bezeichnet), die vom 23. bis zum 27. Juni 1997 in New York tagte, unterbreitet. Das erste Dokument vermittelt einen Überblick über die bisherigen Realisierungen, das zweite mit dem Titel «Strategie» führt die zusätzlichen Massnahmen zur Umsetzung der Politik der nachhaltigen Entwicklung an. Die Sondersitzung der UNO verfolgte das Ziel, eine Bilanz der Fortschritte zu ziehen und die Prioritäten für die kommenden Jahre festzulegen. Dieser wichtige Prozess der regelmässigen Evaluationen ist in den Folgearbeiten internationaler Konferenzen einmalig. Im allgemeinen werden die Ergebnisse von «Rio +5 » freilich als sehr enttäuschend beurteilt. Die Schweiz beteiligte sich daneben auch aktiv an den Konferenzen über die Konventionen, die in Rio unterzeichnet und vom Parlament ratifiziert wurden (Klima und biologische Vielfalt).

Die schweizerischen Nichtregierungsorganisationen in den Bereichen Umwelt und Entwicklung führten während des Jahres 1997 Informationskampagnen zur Konkretisierung der nachhaltigen Entwicklung durch.

\subsection{FÜNF JAHRE NACH DEM ERDGIPFEL}

$\square$ Folgeprozess von Rio in der Schweiz, Vorbereitung für «Rio + 5 »

An der Konferenz der Vereinten Nationen für Umwelt und Entwicklung im Juni 1992 erstellte die Staatengemeinschaft ein politisches und institutionelles Programm, das in den kommenden Jahren als Wegweiser zur Bekämpfung der globalen Umweltprobleme und zur Erreichung einer nachhaltigen Entwicklung dienen soll (Agenda 21).

Der Erdgipfel hat in der Schweiz zu einer Neuausrichtung der Umweltpolitik geführt. War die Umweltpolitik in der Vergangenheit stark auf Gebote und Verbote, in gewissen Bereichen zusätzlich auf Subventionen und Beiträge ausgerichtet, so fand in den letzten Jahren eine vermehrte Hinwendung zu ökonomischen Instrumenten und eine stärkere Integration von Umweltpolitik in andere 
Politikbereiche statt. ${ }^{1}$ Die Umsetzung der Agenda 21 in der Schweiz trägt zur Umorientierung der Umweltpolitik, zur Internalisierung und Globalisierung solcher Massnahmen bei.

Seit der UNCED hat die Schweiz eine Reihe von Berichten verfasst und eine doppelte institutionelle Struktur eingerichtet: verwaltungsintern mit dem IDARio; verwaltungsextern mit Einbeziehung externer Kreise, einer Verbindungsgruppe und 7 Arbeitsgruppen. Diese Struktur sollte die Arbeit besser koordinieren und den Informationsaustausch erleichtern (siehe Kasten «Rio-Folgeprozess 1992 - 1997»).

Im April 1997 - vier Jahre nach der Einsetzung dieser Struktur - hat der Bundesrat das Mandat des IDARio verlängert. Die Verbindungsgruppe jedoch wurde aufgelöst und soll durch einen Rat für nachhaltige Entwicklung (siehe unten) ersetzt werden. Aufgelöst wurden auch drei Arbeitsgruppen (Technologietransfer und technische Zusammenarbeit, Lenkungswirkung und Internalisierung externer Kosten, Kohärenz der Gesetzgebung und der Subventionen). Ein einziger Bericht zum Technologietransfer wurde zwar veröffentlicht ${ }^{2}$, erhielt aber laut Andràs November, Mitglied der Arbeitsgruppe, «nie die gebührende Aufmerksamkeit (...), die Bundesverwaltung liess sich mit der Erstellung einer Studie reichlich Zeit, zumal eine einheitlich definierte Politik nicht den Ansichten aller Bundesämter entsprechen und ihre Divergenzen bei der Politik des Transfers umweltfreundlicher Technologien zutage fördern könnte » ${ }^{3}$. Vier Arbeitsgruppen - Klima, biologische Vielfalt, Wald und das Forum Handel und Umwelt - setzen ihre Sitzungen fort.

\section{$\square$ Nachhaltige Entwicklung in der Schweiz - Strategie des Bundesrates}

Der Bundesrat nahm am 28. Februar 1996 die erste Fassung des Berichts «Nachhaltige Entwicklung in der Schweiz» des IDARio zur Kenntnis und beauftragte gleichzeitig den IDARio, einen Aktionsplan für die nachhaltige Entwicklung der Schweiz auszuarbeiten. Mit dieser Aufgabe wurden sieben Persönlichkeiten betraut, die im «Conseil du développement durable» zusammenarbeiteten ${ }^{4}$. Das Gremium legte im Januar 1997 einen Bericht mit dem Titel «Nachhaltige Entwicklung - Aktionsplan für die Schweiz» vor. Auf dieser Basis erarbeitete der IDARio die Strategie «Nachhaltige Entwicklung in der Schweiz».

Im April 1997 wurden zwei Dokumente veröffentlicht, die als Grundlage der Evaluation und des Aktionsplans für eine der Nachhaltigkeit verpflichtete Schweiz dienen :

- «Nachhaltige Entwicklung in der Schweiz, Stand der Realisierung», Bericht des IDARio, aktualisiert im April 1997;

口 «Nachhaltige Entwicklung in der Schweiz, Strategie», Bundesrat.

Die beiden Dokumente ergänzen einander. Das Dokument «Stand der Realisierung» erläutert das Konzept der nachhaltigen Entwicklung, beschreibt die zu erfüllenden Anforderungen und definiert die Hauptstrategien, welche die Schweiz umsetzen soll. Zudem versucht der Bericht, eine Bilanz der laufenden

1. Philippe Roch, «Umweltpolitik, Eine neue Dimension nach Rio?», Die Volkswirtschaft, 12/1996.

2. IDARio, «Transfer and cooperation in the Area of environmentally sound technologies», Final report of the IDC Rio Working Group, «Technology transfer»/Cooperation, Bern, September 1995.

3. in Jahrbuch Schweiz - Dritte Welt 1997, S. 260.

4. Der «Conseil du développement durable » bestand aus dem Präsidenten Ernst Blaser und sechs weiteren Mitgliedern: Fritz Fahrni, Jacques Forster, Charles Kleiber, Jakob Nüesch, Anne Petitpierre, Roman Rudel. 
Projekte und der jüngsten Beschlüsse zu ziehen, welche bereits zur nachhaltigen Entwicklung in der Schweiz beitragen. Darunter werden erwähnt:

๖ das Programm «Energie 2000» und die am 17. März 1997 unterbreitete Botschaft zum Bundesgesetz über die Reduktion der $\mathrm{CO}_{2}$-Emissionen;

口 die Verkehrspolitik des Bundesrates (Botschaft vom 26. Juni 1996), welche die Attraktivität der Schiene gegenüber der Strasse steigern und eine bessere Internalisierung der externen Kosten erzielen soll;

๖ die Agrarreform, dank welcher ökologische Leistungen wie integrierte Produktion und biologischer Landbau den Landwirten besser abgegolten werden können.

JSDW 1996, Rio-Folgeprozess in der Schweiz, S. 51 - 52.

\section{NACHHALTIGE ENTWICKLUNG: DEFINITION UND INTERPRETATION}

«achhaltige Entwicklung ist eine Entwicklung, welche die heutigen Bedürfnisse zu decken vermag, 《/ ohne für künftige Generationen die Möglichkeit zu schmälern, ihre eigenen Bedürfnisse zu decken " (Definition der Brundtland-Kommission).

Die Solidarität unter der heutigen Generation und gegenüber den künftigen Generationen bildet ein wesentlicher Aspekt der nachhaltigen Entwicklung. Dabei muss sowohl das Gleichgewicht zwischen den Generationen sowie innerhalb einer Generation berücksichtigt werden. Beide Aspekte sind wichtig, zumal sie miteinander verknüpft sind.

Nach der Definition des IDARio beruht die nachhaltige Entwicklung auf drei als gleich wichtig bewerteten Voraussetzungen: ökologische, soziale und ökonomische Aspekte*.

Ökologische Sichtweise : Aus ökologischer Sicht können aus dem Begriff der nachhaltigen Entwicklung die folgenden Thesen und Handlungsgrundsätze abgeleitet werden:

- Die Schadstoffemissionen dürfen nur so hoch sein, dass der Abbau und die Umwandlung dieser Stoffe in unschädliche Substanzen von der Natur bewältigt werden können.

口 Die Natur ist in ihrer ganzen Vielfalt zu erhalten. Beeinträchtigungen sind mit Massnahmen zu kompensieren, welche den Fortbestand von Ökosystemen und die Wahrung der Artenvielfalt gewährleisten.

- Erneuerbaren Ressourcen darf pro Zeiteinheit nicht mehr Substanz entnommen werden, als wieder nachwachsen kann.

- Nicht erneuerbare Ressourcen, wie z.B. fossile Energieträger, dürfen nicht rascher abgebaut werden, als gleichzeitig neue regenerierbare Quellen für deren Ersatz bereitgestellt werden können.

Soziale Sichtweise : Au sozialer Sicht steht die Armut von weltweit gegen einer Milliarde Menschen im Vordergrund, die ihre elementaren Bedürfnisse nicht decken können. Das immer noch anhaltende Bevölkerungswachstum, die Migration der Bevölkerung in die Städte, über die Landesgrenzen hinweg und in andere Kontinente gefährden vielerorts den sozialen Frieden. Dieser Entwicklung kann nur mit besseren sozialen Bedingungen, vor allem bezüglich der Rolle der Frau, begegnet werden. Dazu gehören Verbesserungen der Wohnverhältnisse, der medizinischen Grundversorgung, der Ausbildung und der Beschäftigung. Aus sozialer Sicht sind deshalb diese Probleme vorrangig zu mildern.

Wirtschaftliche Sichtweise : Aus wirtschaftlicher Sicht ist Wachstum eine Notwendigkeit, um den stetig steigenden Bedürfnissen einer wachsenden Weltbevölkerung entsprechen zu können. Analog zu der ökologischen Sichtweise gilt, dass unsere Gesellschaft von den Erträgen und nicht vom Kapitalstock leben sollte. Einnahmen und Ausgaben der privaten und öffentlichen Haushalte und der Unternehmen müssen bei nachhaltigem Wirtschaften langfristig im Gleichgewicht sein, um auch den künftigen Generationen die gleichen wirtschaftlichen Chancen zu sichern.

Aus "Nachhaltige Entwicklung, Aktionsplan für die Schweiz», Conseil du développement durable.

* Nachhaltige Entwicklung in der Schweiz, Bericht des IDARio, Februar 1996, S. 9 - 11. 
Juni 1992

1. März 1993

Frühling 1993

Herbst 1993

Herbst 1994

28. Februar 1996

18. März 1996

Juni 1996

März 1997

23. bis 27. Juni 1997

1998

\author{
Konferenz der Vereinten Nationen für Umwelt und Entwicklung \\ (UNCED), Rio de Janeiro \\ "UNCED 1992, Bericht der Schweiz», BUWAL, April 1992.
}

\title{
Einleitung des IDA-Rio-Prozesses
}

Der Bundesrat überträgt die Koordination der UNCED-Folgearbeiten und die Umsetzung der Agenda 21 dem interdepartementalen Ausschuss IDARio, zu dem Direktoren von 17 Bundesämtern gehören. Der Vorsitz des Ausschusses wird in jährlichem Wechsel von der DEZA, dem BAWI und dem BUWAL wahrgenommen. 1997 führt Philippe Roch, Direktor des BUWAL, den Vorsitz über den IDARio.

Einsetzung einer Kontaktgruppe und sieben Arbeitsgruppen (biologische Vielfalt, Wälder, Klima, Technologietransfer und technische Zusammenarbeit, Lenkungswirkung und Internalisierung der externen Kosten, Kohärenz von Gesetzgebung und Subventionen, Forum Handel und Umwelt).

Die Arbeitsgruppen, deren Mitglieder aus der Bundesverwaltung und aus nichtstaatlichen Bereichen stammen (Umwelt- und Entwicklungs-NRO, Wirtschaft, Akademiker), bilden eine neuartige Arbeitsmethode.

Parlamentsbeschluss für die Ratifizierung der Konvention über die Klimaänderungen.

Parlamentsbeschluss für die Ratifizierung der Konvention über die biologische Vielfalt, wobei allerdings in einer auslegenden Erklärung die Bedeutung der Einhaltung und der Regeln des geistigen Eigentums betont wird.

Der Bundesrat nimmt den Bericht "Nachhaltige Entwicklung in der Schweiz" zur Kenntnis und beauftragt den IDARio, einen Aktionsplan zur nachhaltigen Entwicklung auszuarbeiten.

Der Bundesrat verabschiedet den Bericht über die Legislaturplanung 1995 1999. Erstmals wird die Notwendigkeit für die Schweiz angeführt, das Konzept der nachhaltigen Entwicklung zu konkretisieren.

Der IDARio beauftragt den "Conseil du développement durable", eine Arbeitsgruppe von sieben Personen, mit der Ausarbeitung eines Aktionsplans. Der Bericht "Nachhaltige Entwicklung - Aktionsplan für die Schweiz" diente dem IDARio als Grundlage für seine Strategie "Nachhaltige Entwicklung in der Schweiz".

Der Bundesrat nimmt den Bericht des IDARio "Nachhaltige Entwicklung in der Schweiz - Stand der Realisierung" zur Kenntnis und veröffentlicht die Strategie zu einer "Nachhaltigen Entwicklung in der Schweiz".

Die beiden Dokumente wurden anlässlich der UNGASS vorgestellt.

Sondersitzung der Generalversammlung der Vereinten Nationen zum Stand der Umsetzung der Agenda 21.

Diskussion zum Aktionsplan der Schweiz und zum Konzept der nachhaltigen Entwicklung im Parlament für 1998 geplant.

\section{Internationale Zusammenarbeit}

Die Schweiz setzt ihre internationalen Aktivitäten in verschiedenen Gremien fort:

- Tagungen der Kommission für nachhaltige Entwicklung jedes Jahr im Frühling in New York;

口 Konferenzen der Vertragsparteien der Konventionen über die biologische Vielfalt bzw. über die Klimaänderungen, verschiedene Zwischensitzungen;

口 Sitz im Exekutivrat der GEF, finanzielle Beteiligung an der Fazilität in Höhe von 64 Millionen Franken. 
Die Strategie des Bundesrates mit dem Titel «Nachhaltige Entwicklung in der Schweiz» zielt darauf ab, die nachhaltige Entwicklung in alle Politikbereiche des Bundes einzubeziehen. Genauer bezeichnet die Strategie sieben Aktionsfelder für zusätzliche Massnahmen. Die Bestimmungen beziehen sich auf folgende acht Bereiche :

- Internationales Engagement, Energie, Wirtschaft, Konsumverhalten, Sicherheitspolitik, Ökologische Steuerreform, Bundesausgaben, Umsetzung und Erfolgskontrolle.

Das internationale Engagement verfolgt das Ziel, «einen solidarischen und aktiven Beitrag der Schweiz an die internationalen Bemühungen zur nachhaltigen Entwicklung zu leisten». Weiter heisst es: «Das vermehrte Engagement dient auch unserer Wirtschaft, denn diese profitiert davon, wenn sich die Volkswirtschaften der armen Länder nachhaltig entwickeln, und zieht zusätzlichen Nutzen aus der Verschärfung internationaler Umweltschutzstandards » 5 .

Eine Auswertung der nachhaltigen Entwicklungspolitik wird als unverzichtbar betrachtet. Zu diesem Zweck soll im Laufe des Jahres 1998 ein neuer unabhängiger Rat für nachhaltige Entwicklung als beratendes Organ des Bundesrates eingesetzt werden.

Die beiden Dokumente wurden anlässlich der Sondersitzung der Generalversammlung der Vereinten Nationen vom 23. bis zum 27. Juni 1997 in New York vorgelegt.

\section{Reaktion der Umwelt- und Entwicklungsorganisationen}

Die wichtigsten Umwelt- und Entwicklungsorganisationen ${ }^{6}$ begrüssen die Absichten des Bundesrates, der die erste «Strategie für eine nachhaltige Entwicklung» vorlegt, und unterstützen die Schaffung eines Rates für nachhaltige Entwicklung. Sehr kritisch jedoch äussern sie sich zu den Mitteln, die der Bundesrat für die Umsetzung vorgesehen hat, und beanstanden namentlich das Fehlen überprüfbarer Ziele. Die NRO betonen, dass das Zögern des Bundesrates sich vor allem im Vorschlag zeige, weitere Studien durchzuführen, obwohl die Grunddaten doch bekannt seien und Aktionsmethoden heute auf politischer, nicht auf Expertenebene, definiert werden müssten. Sie verweisen auf die Resignation des Bundesrates in der öffentlichen Entwicklungshilfe (trotz der Zusage der Schweiz, die Hilfe auf 0,4\% des BSP anzuheben, stagniert sie bei 0,3\%), auf den mangelnden Ehrgeiz bei der Steuerreform (Vorschlag, verschiedene Szenarien bis zum Jahr $2001 \mathrm{zu}$ untersuchen) und bei der Senkung der $\mathrm{CO}_{2}$-Abgaben bis zum Jahr 2001 (der Bundesrat hält am Reduktionsziel von 10\% fest, obwohl der Entwurf eines Aktionsplans eine Senkung von $20 \%$ vorgesehen hatte).

Allgemein fehlt nach Auffassung der Organisationen im Rio-Folgeprozess und in der Umsetzung der Agenda 21 ein politischer Wille. Die NRO erinnern daran, dass ein erster Statusbericht erst nach vier Jahren veröffentlicht wurde und der Bundesrat eine eigentliche Kommission für die nachhaltige Entwicklung erst nach fünf Jahren eingesetzt hatte. Ferner vertreten sie die Meinung, dass die Zusammenarbeit zwischen Verwaltung und Zivilgesellschaft von einer echten

5. BUWAL, Der Weg zur nachhaltigen Schweiz, Bern, 1997, S. 24.

6. Pressemitteilung der Umweltorganisationen (SGU, VCS, WWF Schweiz und Pro Natura), April 1997, und Pressekonferenz der Arbeitsgemeinschaft vom 24. April 1997, «5 Jahre nach Rio: Wie zukunftsfähig ist die Schweiz?». 
Partnerschaft noch weit entfernt ist. Sie betonen, dass die Schweiz der Thematik geringe Bedeutung beimesse; das zeige sich unter anderem darin, dass das Parlament sich erst nach der Sondertagung der Generalversammlung zum fünften Jahrestag des Erdgipfels erstmals mit der nachhaltigen Entwicklung auseinandergesetzt hat.

\section{$\square$ Kommission für nachhaltige Entwicklung (CSD-5)}

Die fünfte Tagung der Kommission für nachhaltige Entwicklung (CSD-5) ${ }^{7}$ vom 8. bis zum 25. April 1997 am Sitz der UNO widmete sich den letzten Vorbereitungen der Sondertagung der Generalversammlung der Vereinten Nationen vom Juni 1997 über den Stand der Umsetzung der Agenda 21 (siehe unten). Der Ägypter Mostafa Tolba wurde zum Präsidenten der Sitzung ernannt. Frau Monika Linn Locher vom BUWAL wurde als Vizepräsidentin für die westeuropäische Gruppe eingesetzt.

Die Schweizer Delegation stand unter der Leitung von BUWAL-Direktor Philippe Roch. Mitarbeiter des BUWAL, der DEZA, der Politischen Abteilung V des EDA, des BIGA, des BAWI und der Vertretung in New York waren anwesend. Die Umwelt- und Entwicklungsorganisationen vertrat Rosemarie Bär (Arbeitsgemeinschaft der Hilfswerke).

$\mathrm{Zu}$ Tagungsbeginn fand ein «high level segment»(ministerieller Teil) statt, wobei Minister, Botschafter und NRO ihre Erklärungen vortrugen. Eine Reihe von Dialogen mit den wichtigsten gesellschaftlichen Gruppen (Jugendliche, Wissenschaftler, Frauen, Gewerkschaften, einheimische Völker usw.) fand parallel zu den Sitzungen statt, in denen der eigentliche Konferenztext verhandelt wurde, den die Generalversammlung anschliessend verabschieden sollte. Dieser Text zieht die Bilanz der Fortschritte seit Rio und definiert die Sektoren mit dringlichem Aktionsbedarf. Daneben leiteten der Präsident der CSD-5 Mostafa Tolba und die Vizepräsidentin Monika Linn Locher Konsultationen zum politischen Erklärungsentwurf ein, welcher den Staats- und Regierungschefs bei «Rio +5 » zur Unterschrift vorgelegt werden sollte. Dieser informelle Ansatz wurde an der Schlusssitzung im Plenum von bestimmten Delegationen der G77 kritisiert.

Verschiedene wichtige Punkte (politische Erklärung, Frage der Verabschiedung eines Übereinkommens über Wälder, Aufstockung der Finanzressourcen) blieben letztlich ungelöst. Die Diskussionen wurden im Rahmen von «Rio + 5» wieder aufgenommen. Allgemein verliefen die Verhandlungen schwieriger als erwartet. Einige Delegierte betonten das Unvermögen der CSD, Zielvorgaben und Zeitpläne zu einer besseren Verwirklichung der nachhaltigen Entwicklung vorzugeben, und stellten fest, dass letztlich die Auslegung der Agenda 21 eher eingeengt als ausgebaut wurde. Für die Entwicklungsländer führten die seit 1992 rückläufige öffentliche Entwicklungshilfe und die Versuche, die Last der internationalen Finanzierung der nachhaltigen Entwicklung auf privatwirtschaftliche Investitionen abzuwälzen - ein von den Industrieländern verfochtener Standpunkt -, zu einer Diskreditierung des Konzepts der nachhaltigen Entwicklung ${ }^{8}$.

7. Die vollständige Zusammenfassung der CSD-5 kann im Internet eingesehen werden: Earth Negotiations Bulletin, http ://www. iisd. ca/linkages/

8. In Summary of the CSD-5, http://www. iisd. ca/linkages/ 


$$
\text { «io }+5 »
$$

Mit dem Ziel, fünf Jahre nach dem Erdgipfel von Rio auf höchster Ebene eine erste Bilanz zu ziehen, hat die UNO eine ausserordentliche Generalversammlung einberufen. Die Schweiz war als Nichtmitglied der UNO mit Beobachterstatus dabei.

\section{SONDERSITZUNG DER GENERALVERSAMMLUNG DER VEREINTEN NATIONEN 1997}

\section{Ort und Zeitpunkt}

Die ausserordentliche Generalversammlung tagte in der Woche vom 23. zum 27. Juni 1997 am Sitz der UNO in New York.

Ziele

Zwischenbilanz zur Verwirklichung der nachhaltigen Entwicklung ziehen; konkrete Massnahmen für die nächsten Jahre festlegen.

Bilanz

Die Ergebnisse der Tagung werden allgemein als äusserst dürftig bewertet. Es wurde ein rund fünfzig Seiten starkes "Umsetzungsprogramm" angenommen. Anders als geplant verabschiedete die Versammlung jedoch keine politische Erklärung, sondern einen als "Engagement" bezeichneten Konsenstext. So blieb de facto ein klares politisches Signal für den Schutz von Klima und Wäldern aus.

\section{Teilnehmer}

60 Staats- und Regierungschefs und zahlreiche Minister reisten zu der Veranstaltung an. Zudem waren die nationalen Delegationen von über 164 Staaten präsent.

\section{Schweizerische Delegation}

Die schweizerische Delegation stand unter der Leitung von Bundesrätin Ruth Dreifuss. Mitarbeiter des BUWAL, der DEZA, der Politischen Abteilung V des EDA, des BIGA sowie die ständige Beobachtermission der Schweiz bei den Vereinten Nationen waren anwesend. Umwelt- und Entwicklungsorganisationen waren durch die Arbeitsgemeinschaft der Hilfswerke vertreten.

Kontaktperson für die Verwaltung

Monika Linn Locher, BUWAL

\section{$\square$ Ministertreffen}

Staatschefs, Minister und Staatssekretäre, Vertreter internationaler Organisationen und NRO trugen 187 Erklärungen zur Evaluation der Umsetzung der Agenda 21 vor'. Die Redner hielten generell fest, dass die Grundsätze der Agenda 21 zur Zeit in nationalen Gesetzen verankert werden und dass zwei in Rio unterzeichnete Übereinkommen (biologische Vielfalt und Klima) sich in Anwendung befinden. Allerdings wurde in zahlreichen Wortmeldungen hervorgehoben, dass die Verbrauchs- und Produktionsmuster trotz der Verpflichtungen von Rio auf Niveaus blieben, die mit der Nachhaltigkeit nicht zu vereinbaren sind. Die Abholzung geht weiter, und auch in zentralen Bereichen wie Klimawandel und Trinkwasserbewirtschaftung wird ein politischer Wille vermisst. Schliesslich verfügen zahlreiche Entwicklungsländer noch immer nicht über die wesentlichen umweltschonenden Technologien. Daneben wurde auch unterstrichen, dass die öffentliche Entwicklungshilfe weiterhin schrumpft und durch eher auf ökonomische als ökologische Ziele ausgerichtete - Auslandsinvestitionen ersetzt wird. 
In ihrer Botschaft stellte Bundesrätin Ruth Dreifuss, Vorsteherin des Eidgenössischen Departements des Innern, kritisch fest, viele Hoffnungen seien heute enttäuscht worden. Als besonders vernichtende Niederlage bezeichnete sie die Bekämpfung der Armut. Die Bundesrätin ging daneben auf verschiedene von der Schweiz vertretene Punkte ein. Was die Zusammenarbeit mit den Ländern des Südens angeht, so könne die Schweiz als gebirgiges Land einen wichtigen Beitrag leisten ${ }^{10}$. Ruth Dreifuss plädierte für eine bessere Preisinternalisierung der sozialen und ökologischen Kosten. In dieser Hinsicht setzt sich die Schweiz für die Einführung einer Abgabe auf Flugzeugkerosin ein. Schliesslich betonte sie, dass die Schweiz die Neuaufstockung der Globalen Umweltfazilität unterstützt und schlug vor, diese als ständigen Finanzierungsmechanismus der Konventionen über Klimaänderungen und Artenvielfalt einzusetzen.

\section{$\square$ Verpflichtungserklärung}

Die 164 Staaten konnten sich nicht auf eine endgültige politische Erklärung einigen. Meinungsunterschiede lösten unter anderem folgende Fragen aus: Definition der nachhaltigen Entwicklung, Termine der Verpflichtungen, Mittelzuweisung für die öffentliche Entwicklungshilfe, Mobilisierung interner Ressourcen. Die Entwicklungsländer verweigerten ihre Unterstützung mit dem Argument, dass die Industrieländer weder Finanz- noch Technologietransfers vorantrieben. Die Industrieländer ihrerseits wollten sich unter keinen Umständen auf einen Zeitplan und auf konkrete Verpflichtungen festlegen. Da keine Einigung zu einer politischen Erklärung der Staatschefs erzielt wurde, verabschiedete die für die Redaktion zuständige Kommission statt dessen eine Verpflichtungserklärung von weit geringerer politischer Tragweite, die zwar einige positive Ergebnisse anerkennt, aber doch zum Schluss kommt, dass sich der Umweltzustand in den letzten fünf Jahren weiter verschlechtert hat.

\section{$\square$ Programm der weiteren Umsetzung von Agenda 21}

In diesem Dokument von rund fünfzig Seiten werden die Arbeiten der fünften Kommission für nachhaltige Entwicklung und der ausserordentlichen Generalversammlung der UNO zusammengefasst. Die Verpflichtungserklärung dient als Einführung, gefolgt von drei grossen Kapiteln: Evaluation der seit der UNCED verwirklichten Fortschritte, Umsetzung in Sektoren mit dringendem Handlungsbedarf, internationale institutionelle Vereinbarungen. Der Anhang enthält das Arbeitsprogramm der Kommission für nachhaltige Entwicklung für die Jahre 1998 - 2002.

Die Hauptpunkte des Programmes sind:

\section{Erwärmung der Erdatmosphäre}

Es konnte kein Konsens für einen Aktionsplan gegen die Erwärmung der Erdatmosphäre erzielt werden. Die europäischen Länder wollten anlässlich der Sitzung ein klares politisches Signal an die Klimakonferenz senden, welche im Dezember 1997 in Japan tagte, und ihre Bereitschaft bekräftigen, die $\mathrm{CO}_{2}$-Emissionen bis zum Jahr 2010 um 15\% zu verringern. Die Vereinigten Staaten lehnten es ab, sich auf eine Grenzzahl festzulegen.

10. Die Schweiz organisierte am Rande der Sondersitzung ein Seminar, auf dem das von der DEZA, der Universität Bern und der FAO in Zusammenarbeit produzierte Werk über die Bergregionen vorgestellt wurde. 


\section{Schutz der Wälder}

Die Diskussionen zu diesem Thema erwiesen sich als sehr problematisch. Die Europäische Union, Kanada und Costa Rica (im Namen Zentralamerikas) befürworteten die Aufnahme von Verhandlungen für eine Waldkonvention. Die Vereinigten Staaten, Japan, die G77 und China insbesondere waren jedoch dagegen. Schliesslich wurde mit der Schaffung eines zwischenstaatlichen Forums über Wälder, das die Beschlüsse der zwischenstaatlichen Expertengruppe umsetzen und mögliche Konventionsinhalte erarbeiten soll, eine Konsensentscheidung gefunden.

\section{Entwicklungshilfe}

Die in Rio abgegebenen Versprechen, die Entwicklungshilfe zu erhöhen, wurden nicht eingelöst. Die Diskussionen zu diesem äusserst umstrittenen Thema mündeten in der Anregung, die öffentliche Entwicklungshilfe solle zwar die wichtigste Finanzierungsquelle der Entwicklungsländer bleiben, aber gleichzeitig als Katalysator für die Mobilisierung privater Ressourcen wirken.

\section{$\square$ Standpunkt der Schweiz}

Der Standpunkt der Schweiz wurde im allgemeinen von Ruth Dreifuss im ministeriellen Teil vertreten (siehe unten). Die Schweiz betonte insbesondere die soziale Dimension der nachhaltigen Entwicklung zugunsten der Schaffung von Arbeitsplätzen und erinnerte an die Verpflichtungen des Weltgipfels für soziale Entwicklung von Kopenhagen (1995). Da die Schweiz nicht Mitglied der Vereinten Nationen ist, konnte sie sich zwar als Beobachterin, aber ohne Stimmrecht, an den Diskussionen beteiligen. Dessen ungeachtet war mit Monika Linn Locher, Sektionschefin in der Abteilung Internationales des BUWAL, eine Schweizerin zur Vizepräsidentin der fünften Kommission für nachhaltige Entwicklung ernannt worden.

\section{$\square$ Auswertung des «Erdgipfels + 5》}

Nach Auffassung der NRO und der Medien sind die Ergebnisse von «Rio + 5» dürftig, was indessen nach den schwierigen Verhandlungen zur Vorbereitung der Konferenz (CSD-5) abzusehen war. Das politische Scheitern der ausserordentlichen UNO-Versammlung wiegt schwer, denn eine klar gezeigte Bereitschaft der Regierungen zur Umsetzung von Agenda 21 wäre unverzichtbar gewesen, um die Initiativen zu koordinieren und die Zivilgesellschaft zur Mitwirkung zu motivieren.

\section{BERICHT DES UMWELTPROGRAMMES DER VEREINTEN NATIONEN " GLOBAL ENVIRONMENT OUTLOOK"}

nlässlich des Gipfels "Rio + 5 » zog das UNEP in einem Bericht Bilanz zur Umweltlage des Planeten. A In den Bereichen institutionelle Entwicklung, Stärkung des internationalen Konsens, öffentliche Beteiligung und Aktionen auf Initiative des öffentlichen Sektors wurden einige Fortschritte erzielt. Als Resultat gelang es verschiedenen Ländern, das Tempo der Zerstörung der Ressourcen zu drosseln. Das Bevölkerungswachstum hat sich allgemein verlangsamt, was weitgehend auf die Ausweitung der grundlegenden Schulbildung und Gesundheitsversorgung zurückgeht. Trotz dieser Errungenschaften aber ist eine weitere Verschlechterung der allgemeinen Tendenzen im Gange. Der Anstieg der Verschmutzungswerte droht die Absorptionsfähigkeit der globalen Umwelt zu übersteigen.

Eine englische Version des Berichtes "Global Environment Outlook" kann an folgender Internet-Adresse eingesehen werden: http://www. grid. unep. ch/geo1/index. htm 


\section{$\square$ Konkretisierung des Begriffs nachhaltige Entwicklung}

«Nachhaltig ist eine Entwicklung, wenn sie die heutigen Bedürfnisse zu decken vermag, ohne für künftige Generationen die Möglichkeiten zu schmälern, ihre eigenen Bedürfnisse zu decken» - nach diesem Leitmotiv soll die nachhaltige Entwicklung verwirklicht werden. In der Schweiz hat das BUWAL ein umfassendes mehrjähriges Programm lanciert, um Projekte zu fördern, die zur Umsetzung des Aktionsplans dienen. Zudem haben die Umwelt- und Entwicklungsorganisationen 1997 verschiedene wichtige Beiträge zur Konkretisierung des Konzepts der nachhaltigen Entwicklung geleistet.

«Nord-Süd-Kampagne für Nachhaltige Entwicklung »

Am 30. Januar 1997 startete die Arbeitsgemeinschaft Swissaid/Fastenopfer/Brot für alle/Helvetas/Caritas eine breitangelegte Initiative unter dem Titel «NordSüd-Kampagne für Nachhaltige Entwicklung», die bis 1998 dauern soll und verschiedene Veranstaltungen beinhaltet. Referenzdokument der Kampagne ist das «Nord/Süd-Manifest», das in 21 Thesen zur nachhaltigen Entwicklung die Rolle der Schweiz auf internationaler Ebene und die Handlungsmöglichkeiten verschiedener Akteure definiert: wirtschaftliche und politische Kreise, Zivilgesellschaft und Verbraucher ${ }^{11}$.

Die Arbeitsgemeinschaft führte im Rahmen ihrer Kampagne eine umfassende Untersuchung ${ }^{12}$ bei einer repräsentativen Stichprobe in der Schweiz durch, die ergab, dass ein Drittel (34\%) der Bevölkerung das Konzept nachhaltige Entwicklung richtig deuten konnte. Ein Viertel (25\%) war dazu nicht in der Lage. Mehr als die Hälfte aller Befragten finden, dass die nachhaltige Entwicklung den Norden wie den Süden betreffe und dass die entsprechenden Anstrengungen seitens der Schweizer Behörden, Wirtschaft und Bürger/innen noch ungenügend seien.

«Umwelt - Wirtschaft - Soziales: 18 Thesen zur nachhaltigen Entwicklung»Beitrag der Umweltorganisationen

Die Umweltorganisationen legten eine zweisprachige Broschüre mit dem Titel «Umwelt - Wirtschaft - Soziales: 18 Thesen zur nachhaltigen Entwicklung als Diskussionsbeitrag vor ${ }^{13}$ Anfänglich hatten der VCS, die SGU, WWF Schweiz und Pro Natura ein der nachhaltigen Entwicklung verpflichtetes Reformpaket für die Bundesverfassung ausgearbeitet. Dieses erste Ziel wurde in der Folge nicht aufgegeben, sondern im Sinne einer Ergänzung abgeändert. Die Broschüre enthält eine Reihe von Vorschlägen zu folgenden Themen :

口 Rolle des Staates, Wichtigkeit, das Konzept der nachhaltigen in die Gestaltung der Politik einzubeziehen;

- Wirtschaftliches und Soziales mit besonderer Betonung der Aspekte Arbeit, Einkommen und Fiskalität;

口 Umwelt und Technik: Gefordert werden eine rationelle Nutzung der natürlichen Ressourcen, Schutz von Umwelt und Landschaft, rationelle Raumplanung, weniger Individualverkehr und Warentransporte;

11. Veröffentlichung der Arbeitsgemeinschaft, Süd-Magazin, «Was heisst Nachhaltige Entwicklung?». Die Broschüre vermittelt einen Überblick über die globale Lage, nennt die Ziele der Kampagne und präsentiert das Nord-Süd-Manifest. Erhältlich beim Sekretariat der Arbeitsgemeinschaft, Tel. 021/617 4353.

12. Isopublic-Erhebung, im Auftrag der Arbeitsgemeinschaft durchgeführt, Januar 1997.

13. Pro Natura, SGU, WWF Schweiz, VCS, «Umwelt - Wirtschaft - Soziales: 18 Thesen zur nachhaltigen Entwicklung ", Beitrag der Umweltorganisationen, Mai 1997. 
口 Durchsetzung und Controlling: Normen und staatliches Handeln müssen widerspruchsfrei sein; es soll ein System der Klage gegenüber Exekutive und Legislative eingeführt werden, wenn diese einen Verfassungsauftrag nicht erfüllen.

Am originellsten an der Veröffentlichung sind die Vorschläge zur Rechtsordnung. Im übrigen werden die traditionellen Themen der nachhaltigen Entwicklung aufgegriffen.

\section{«Das Existenz-Maximum - Grundlagen für eine zukunftsfähige Schweiz»}

Ausgehend von alltäglichen Gesten will das Buch der Erklärung von Bern ${ }^{14}$ den Leser zu einem Konsumverhalten im Sinne der nachhaltigen Entwicklung motivieren. Des weiteren behandelt das Werk verschiedene Aspekte der Nord-SüdBeziehungen mit Auswirkungen auf die Umwelt und auf den wirtschaftlichen und sozialen Bereich.

\section{«Comprendre la norme ISO 14'001»}

Die Unternehmen spielen bei der Umsetzung der nachhaltigen Entwicklung eine Schlüsselrolle. Als Fortsetzung des Erdgipfels von Rio schlugen die Wirtschaftskreise die Einführung einer internationalen Norm ISO 14'001 vor, welche 1996 gebilligt wurde. Die Norm ISO 14'001 setzt Leitlinien für die Errichtung eines Umwelt-Managementsystems fest, das einem Unternehmen ermöglicht, die eigenen Auswirkungen auf die Umwelt zu definieren, mögliche Verbesserungen aufzuzeigen und umzusetzen. Die SGU hat als Einleitung zur Norm ISO 14'001 eine Broschüre ${ }^{15}$ veröffentlicht, welche die Voraussetzungen für die Errichtung eines Umwelt-Managementsystems erklärt. Die Zahl der Zertifikate nach ISO 14'001 nimmt weltweit rasch zu. In der Schweiz werden bereits über 100 zertifizierte Unternehmen gezählt (von der Schwerindustrie über Bankinstitute bis zu Kosmetikaherstellern). Die Gültigkeit des Zertifikats ist auf drei Jahre beschränkt. Nach dieser Frist ist eine neue Überprüfung notwendig.

\section{QUELLEN}

Bundesrat, Nachhaltige Entwicklung in der Schweiz - Strategie, Bern, April 1997.

IDARio, Nachhaltige Entwicklung in der Schweiz - Stand der Realisierung, Bericht, aktualisiert im April 1997, Interdepartementaler Ausschuss Rio, Bern, April 1997.

Conseil du développement durable, Nachhaltige Entwicklung - Aktionsplan für die Schweiz, BUWAL, März 1997.

BUWAL, Der Weg zur nachhaltigen Schweiz, Bern, 1997.

BUWAL, Pressemitteilung, «Die Schweiz soll nachhaltiger werden», 14. April 1997.

BUWAL, Umweltschutz, BUWAL-Bulletin, «Nachhaltige Entwicklung - fünf Jahre nach Rio», 2/97.

BUWAL, Umweltschutz, BUWAL-Bulletin, Schweizer Bilanz «Rio plus fünf »: Der Bewusstseinswandel hat eingesetzt, $3 / 97$.

Earth Negotiations Bulletin, Vol. 5, Nr. 82 (Site http://www. iisd. callinkages/).

Arbeitsgemeinschaft, Presseunterlagen, « 5 Jahre nach Rio: Wie zukunftsfähig ist die Schweiz?», 24. April 1997.

Arbeitsgemeinschaft, Swiss Coalition News, «Rio + 5, more than sustainable illusions?», Nr. 12, Mai 1997.

SGU, VCS, WWF Schweiz und Pro Natura, Plan d'action du Conseil fédéral pour le développement durable-trop peu, trop tard et si timide... dommage, Pressemitteilung der Umweltorganisationen, April 1997.

Journal de Genève, 5. April 1997 «Philippe Roch: j'ai appris à comprendre les exigences de l'économie», 23. Juni 1997 «Les Terriens se retrouvent à New York», 28. Juni 1997 «Au sommet de la Terre, l'esprit de Rio est retombé comme un souffle».

La Liberté, 25. Juni 1997 «Pour sauver la Terre, il faut dépasser les divergences politiques», 26. Juni 1997 «Quelle organisation pourrait sauvegarder l'environnement?», 27. Juni 1997 «Le Sommet de la terre menace de capoter sur la question des gros sous».

14. Erklärung von Bern, Das Existenz-Maximum - Grundlagen für eine zukunftsfähige Schweiz, Zürich, 1997. Erhältlich beim Sekretariat der Erklärung von Bern, Tel. 01/271 6434.

15. Schweizerische Gesellschaft für Umweltschutz, Comprendre la norme ISO 14001, SGU, September 1996. Nur in französischer Fassung erhältlich beim Sekretariat der SGU, Tel. 022/329 9929. 
Le Monde, 24. Juni 1997 «L'environnement, source de discorde au sommet du G8», 28. Juni 1997 «L'environnement s'est dégradée depuis cinq ans ».

Neue Zürcher Zeitung, 5. Juni 1997 «Rio-Zwischenbilanz nach fünf Jahren», 24. Juni 1997 «Erdgipfel plus fünf in New York eröffnet», 25. Juni 1997 «Ruth Dreifuss am New Yorker Erdgipfel», 28. Juni 1997 «Selbstkritik der USA am Erdgipfel in New York» und «Fünf Jahre nach Rio - die Bilanz bleibt offen».

Tages-Anzeiger, 28. Juni 1997 «Im Gespräch, Monika Linn Locher zum Umweltgipfel».

INTERNET-ADRESSEN

Offizieller Server der Ausserordentlichen Tagung der UNO-Generalversammlung (Rio + 5): http://www. un. org/DPCS/earthsummit/

Arbeitsgemeinschaft, Süd-Magazin, «Was heisst nachhaltige Entwicklung?», 2/1997.

Arbeitsgemeinschaft, Swiss Coalition News, «North-South Manifesto for sustainable development», Nr. 11, Februar 1997.

Pro Natura, SGU, WWF Schweiz, VCS, «Umwelt - Wirtschaft - Soziales: 18 Thesen zur nachhaltigen Entwicklung», Beitrag der Umweltorganisationen, Mai 1997.

Erklärung von Bern, Das Existenz-Maximum - Grundlagen für eine zukunftsfähige Schweiz, Zürich, 1997.

Schweizerische Gesellschaft für Umweltschutz, Comprendre la norme ISO 14001, SGU, September 1996.

\subsection{SCHUTZ UND NACHHALTIGE BEWAHRUNG DER BIOLOGISCHEN VIELFALT}

Auf menschliches Einwirken zurückgehende Aktivitäten stellen eine immer grössere Bedrohung für die biologische Vielfalt (Tier- und Pflanzenarten, genetisches Material und ihre Ökosysteme) dar. Die Konzentration der Verschmutzung, die nicht nachhaltigen landwirtschaftlichen Praktiken, massive Abholzungen und Klimaänderungen gehören zu den Faktoren, welche das Ökosystem beeinträchtigen, Lebensräume zerstören und so das Schwinden der Artenvielfalt mit verursachen.

\section{INTERNATIONALE ÜBEREINKOMMEN ZUM SCHUTZ DER BIOLOGISCHEN VIELFALT}

• Internationale Konvention zur Regelung des Walfangs vom 2. Dezember 1946

口 Internationale Übereinkunft zum Schutze der Vögel vom 18. Oktober 1950

口 Übereinkommen zum Schutz von Feuchtgebieten von internationaler Bedeutung, insbesondere als Lebensraum für Watt- und Wasservögel vom 2. Februar 1971 (Ramsar-Konvention)

ـ Übereinkommen über den internationalen Handel mit gefährdeten Arten freilebender Tiere und Pflanzen vom 3. März 1973 (CITES)

• Übereinkommen zur Erhaltung der wandernden wildlebenden Tierarten vom 23. Juni 1979

口 Übereinkommen über die Erhaltung der europäischen wildlebenden Pflanzen und Tiere und ihre natürlichen Lebensräume (Konvention von Bern)

口 Konvention über die biologische Vielfalt vom 5. Juni 1992 (Rio-Konvention)

\section{$\square$ Dritte Konferenz der Vertragsparteien der Konvention über die biologische} Vielfalt (COP-3)

Im Anschluss an eine Reihe rechtlicher Vereinbarungen über den Schutz der Arten und der Lebensräume stellt die Konvention über die biologische Vielfalt von 1992 das jüngste multilaterale Instrument als Reaktion auf die Umweltkrise dar. Die Konvention ist im Dezember 1993 in Kraft getreten. Ziel ist die Erhaltung der Artenvielfalt, ein nachhaltiger Umgang mit den biologischen Ressourcen und eine gerechte Aufteilung der Gewinne aus der Nutzung der genetischen Ressourcen. Seit Inkrafttreten der Konvention über die biologische Vielfalt haben sich die Staaten zu drei internationalen Konferenzen, den sogenannten 
Vertragsparteienkonferenzen (COP), zusammengefunden. Die COP-1 legte ein mittelfristiges Arbeitsprogramm fest und beschloss, eine besondere Arbeitsgruppe einzusetzen: Das Nebenorgan (vor allem unter dem Kürzel SBSTTA bekannt) soll wissenschaftliche, technische und technologische Beratung erteilen. Die COP-2 einigte sich auf Montreal als Sitz des Konventionssekretariats. Ferner beschloss sie, ein Protokoll zur biologischen Sicherheit als Ergänzung der Konvention auszuarbeiten und die Globale Umweltfazilität (GEF) als vorläufigen Finanzierungsmechanismus zu benutzen. Behandelt wurden zudem die Situation der Biodiversität in Küstengebieten und Meeren sowie die Frage der Wälder als «Reservoir» der Artenvielfalt.

\section{DRITTE KONFERENZ DER VERTRAGSPARTEIEN DER KONVENTION ÜBER DIE BIOLOGISCHE VIELFALT - COP 3}

Ort und Zeitpunkt Die Konferenz fand vom 4. - 15. November 1996 in Buenos Aires statt.

Hauptthemen Diskussionen zu den Empfehlungen der zweiten Tagung des SBSTTA; Auswertung des Informationsaustausch-Mechanismus; Finanzressourcen und Finanzierungsmechanismus (GEF); allgemeine Massnahmen zur Bewahrung und zur nachhaltigen Nutzung der Artenvielfalt; Biodiversität in der Landwirtschaft; Arbeitsprogramm zur biologischen Vielfalt der auf dem Land frei vorkommenden Arten; Wissen, Innovationen und Praktiken der lokalen einheimischen Gemeinschaften; Zugang zu genetischen Ressourcen; Technologietransfer; geistiges Eigentum; biologische Sicherheit.

Teilnehmer

Die Delegationen der 160 Unterzeichnerstaaten der Konvention beteiligten sich an den Arbeiten der COP-3. Vom 13. - 14. November 1996 fand eine Ministertagung statt. Regierungen, internationale Organisationen und NRO trugen über 80 Erklärungen vor. Das Wort ergriffen unter anderen die Präsidentin der COP-3, Maria Julia Alsogaray, die Exekutivdirektorin der UNCED, Elizabeth Dowdeswell, sowie der argentinische Präsident, Carlos Menem.

Schweizerische Delegation Die schweizerische Delegation in der COP-3 wurde von BUWALDirektor Philippe Roch geleitet. Ihre Mitglieder waren Louis Curat (DEZA), der mit dem Vizevorsitz der Konferenz beauftragt wurde, Robert Lamb und François Pythoud (BUWAL), Jürgen Benz (DEZA), Benno Bättig (BAWI) und Lorenza Ferrari (EIGE).

Kontaktperson für die Bundesverwaltung Philippe Roch, BUWAL

In der dritten Vertragsparteienkonferenz (COP-3) wurden die Funktionsweise der Konferenz, die Rolle und die Beziehungen $\mathrm{zu}$ bestehenden internationalen Instrumenten insbesondere in den Bereichen geistiges Eigentum, Wälder und Agenda 21 genauer ausgestaltet. In diesem Prozess konnte die Vertragsparteienkonferenz allmählich an Profil gewinnen. So bemühte sie sich, ihre Autorität über die GEF und ihre Autonomie gegenüber dem UNED zu sichern. Daneben wurden das Arbeitsprogramm und der künftige Aktionsplan verfeinert. Während der Konferenz ergriffen die Delegierten mehrere wichtige Massnahmen: Ausarbeitung eines Arbeitsprogramms zur Artenvielfalt in der Landwirtschaft und eines etwas bescheideneren Programms zur Biodiversität im Forstbereich; ein in 
langwierigen Verhandlungen erarbeitetes «Memorandum of Understanding » mit der GEF; Organisation eines Workshops über die Kenntnisse, Innovationen und Praktiken der lokalen einheimischen Gemeinschaften in der Zeit zwischen den Tagungen; Antrag auf Beobachterstatus im Ausschuss für Handel und Umwelt der WTO; Vorbereitung einer Erklärung für die Sondersitzung der Generalversammlung der UNO über die Umsetzung der Agenda 21.

Finanzressourcen und Finanzierungsmechanismus

Ein Dokument mit einem Appell an alle multilateralen und bilateralen Finanzierungsinstitutionen, die Forderungen der Konvention verstärkt zu beachten, konnte ohne wesentliche Schwierigkeiten verabschiedet werden.

Dagegen wurden zum «Memorandum of Understanding» zwischen der Vertragsparteienkonferenz und der globalen Umweltfazilität lange Verhandlungen geführt. Die GEF soll noch bis zur nächsten Vertragsparteienkonferenz auf vorläufiger Basis funktionieren. Diskutiert wurden folgende Punkte:

口 Möglichkeiten für die Vertragsparteienkonferenz, als Berufungsinstanz gegen Entscheidungen der GEF zu handeln;

口 Evaluation der «Biodiversitäts »-Projekte, die der globale Umweltfonds finanzieren soll;

口 Diskussion zur Effizienzkontrolle der GEF anlässlich der COP-4;

๑ neue Bereiche, für welche der Bund eine Finanzierung der Umweltfazilität wünscht (Biodiversität in der Landwirtschaft, Taxonomie, biologische Sicherheit, Austauschmechanismus für Fragen des geistigen Eigentums und die Beteiligung einheimischer Bevölkerungen usw.).

In den Diskussionen zu den verschiedenen Punkten zeigten sich die immer noch starken Spannungen zwischen der GEF und der Vertragsparteienkonferenz.

Zusammen mit Argentinien organisierte die Schweiz während der Vertragsparteienkonferenz zwei Seminare über die GEF und eine Reise nach Patagonien, während welcher 60 Teilnehmer ein von der GEF finanziertes Projekt besichtigen konnten. Dieser Besuch trug zum besseren Verständnis der Funktionsweise der GEF und zur Anbahnung eines Dialogs unter den verschiedenen Partnern GEF-Exekutivdirektor, GEF-Mitarbeiter, Vertreter der offiziellen Delegationen und der NRO - bei.

\section{Biodiversität in der Landwirtschaft}

Die Biodiversität in der Landwirtschaft stellte ein vorrangiges Sitzungsthema dar. In diesem Bereich sollen verstärkt Synergien mit den Arbeiten anderer internationaler Foren - insbesondere der FAO - entwickelt und die Berücksichtigung der Konventionsziele in den Agrarpolitiken gefördert werden.

Kenntnisse, Innovationen und Praktiken der lokalen

einheimischen Bevölkerungen

Die Diskussion zu diesem Punkt wurde durch die Präsenz von Vertretern einheimischer Völker aus Lateinamerika, dem Pazifik und Afrika, die von mehreren Entwicklungsländern unterstützt wurde, bereichert. Die Schweiz unterstrich in ihrer Wortmeldung die Schlüsselrolle der autochthonen Bevölkerungen und ihres überlieferten Wissens für die Bewahrung und nachhaltige Nutzung der biologischen Vielfalt und betonte die Notwendigkeit, ein Entschädigungssystem für das 
überlieferte Wissen der einheimischen Bevölkerungen und lokalen Gemeinschaften einzuführen. Zu dieser Thematik wurde im November 1997 eine Arbeitssitzung organisiert, um allgemeine Unterlagen zur Frage vorzubereiten. Eine eingehende Diskussion soll auf einer der nächsten Vertragsparteienkonferenzen stattfinden.

\section{Geistige Eigentumsrechte}

In der Diskussion zu den geistigen Eigentumsrechten zeigte sich die Unzufriedenheit bestimmter Delegationen und bestimmter NRO mit der Behandlung der Thematik im TRIPS-Übereinkommen der WTO. Probleme bereiten folgende Punkte: Fehlen eines geistigen Eigentumssystems zur Anerkennung des überlieferten Wissens; bestimmte Formen der Patenterteilung für biotechnologische Produkte; Rolle der WTO in der universalen Regelschaffung im Bereich des geistigen Eigentums. Im Zusammenhang mit diesem Punkt beschloss die Vertragsparteienkonferenz, einen Beobachterstatus beim Ausschuss für Handel und Entwicklung der WTO zu beantragen.

$\llbracket$ JSDW 1998, Dossier «Analysen und Stellungnahmen».

\section{Erarbeitung eines Protokolls über die biologische Sicherheit}

Die Nutzung des Potentials der modernen Biotechnologie in Sektoren wie Gesundheit, Landwirtschaft oder Umwelt muss in einem globalen Rahmen geregelt werden und eine hohe Sicherheit für Mensch und Umwelt garantieren. In diesem Sinne wurden 1995 Verhandlungen zur Erarbeitung eines Protokolls über die Vorbeugung von Risiken der Biotechnologien eingeleitet. Die Vertragsparteienkonferenz hat vom Stand der Arbeiten einer Ad-hoc-Gruppe über die biologische Sicherheit Kenntnis genommen. Diese Arbeitsgruppe hat den Auftrag, als Ergänzung der Konvention zu diesem Punkt ein Protokoll über die biologische Vielfalt zu erarbeiten. Die Diskussionen zum Protokollentwurf dürften 1998 anlaufen.

\section{Haltung der Schweiz in der COP-3}

Unter den zentralen Konferenzthemen hat die Schweiz besonders die Verbesserung der Beziehungen zwischen der Vertragsparteienkonferenz und dem Finanzierungsmechanismus der Konvention, der globalen Umweltfazilität (Global Environment Facility), hervorgehoben. Ferner beteiligte sie sich aktiv an den Diskussionen zu den Themen Wälder, Sicherheit in der Biotechnologie, traditionelle Kenntnisse der einheimischen Bevölkerungen, Zugang zu genetischen Ressourcen und Gewinnverteilung aus der Nutzung dieser Ressourcen. Insbesondere plädierte die Schweiz für eine verstärkte Mitwirkung des privaten Sektors bei der Entwicklung der Arbeiten zu Wirtschaftsmassnahmen, die zur Bewahrung und zur nachhaltigen Nutzung der Ressourcen beitragen sollen.

In der Erklärung der Schweiz im ministeriellen Teil der COP-3 erwähnte Philippe Roch einige der von der Schweiz aufgegriffenen Punkte und bestand nachdrücklich auf dem Verbesserungsbedarf hinsichtlich der Arbeitsbedingungen der Vertragsparteienkonferenz (klarere Definition der Ziele, Beschränkung der Dokumentenzahl und der mehr oder weniger «formellen» Gruppen, Verlängerung der Arbeitsfristen, um bessere Analysen zu erzielen) ${ }^{16}$.

16. Erklärung der Schweiz, vorgetragen von BUWAL-Direktor Philippe Roch im ministeriellen Teil der dritten Konferenz der Vertragsparteien über die biologische Vielfalt (Buenos Aires, 13. November 1997). 
Die vierte Konferenz der Vertragsparteien wird vom 4. bis zum 15. Mai 1998 in Bratislava in der Slowakei tagen. Einen Kernpunkt der Konferenz werden die Konventionsbestimmungen über die gerechte Aufteilung der Ressourcen bilden. Es handelt sich um ein komplexes und politisch heikles Thema. Zum einen wurden die Bestimmungen noch nicht ausreichend konkretisiert, zum anderen sind sich die Länder des Nordens und des Südens in der Auslegung uneinig (Zugang zu genetischen Ressourcen, Zugang zu Biotechnik, Aufwertung des Wissens der einheimischen Völker und der bäuerlichen Gemeinschaften).

凹SDW 1995, S. 66 - 68 (Ratifizierung der Konvention durch die Schweiz mit einer auslegenden Erklärung der Schweiz)

USDW 1997, S. 55 - 59 (Text der Konvention, COP-1, COP-2)

\section{QUELLEN}

Übereinkommen der Vereinten Nationen über die biologische Vielfalt.

Botschaft über das Übereinkommen der Vereinten Nationen über die biologische Vielfalt.

United Nations Environment Programme, Rapport de la troisième réunion de la Conférence des parties à la convention sur la diversité biologique, UNEP/CBD/COP/3/38, 11. Februar 1997.

BUWAL, Umweltschutz, BUWAL-Bulletin, «Übereinkommen über die biologische Vielfalt - eine Zwischenbilanz», S. 27 $-34,2 / 97$.

BUWAL, Pressemitteilung, «Konferenz der Vertragsparteien über die biologische Vielfalt, Aktive Teilnahme der Schweiz», 15. November 1996.

Earth Negotiations Bulletin, Dritte Tagung der Konferenzparteien des Übereinkommens über die biologische Vielfalt, 4.

- 15. November 1997, vol. 09: 65, verfügbar im Internet: http://www. iisd. ca/linkages/

Ecodécision, «Biodiversité, sociodiversité et aires protégés», Nr. 23, Winter 1997.

Journal de Genève, 5. November 1997 «La Suisse défend la biodiversité en Argentine».

Neue Zürcher Zeitung, 15. Januar 1997 «Wann ist der Schutz der Biodiversität nachhaltig? ».

INTERNET-ADRESSE

Official Web site of the Convention on Biological Diversity:

http://www. biodiv. org/

Earth Negotiations Bulletin (electronic clearing-house for information on past and upcomping internationale meetings related to environment and development:

http://www. iisd. ca/linkages

\section{Übereinkommen über den internationalen Handel mit gefährdeten Arten freilebender Tiere und Pflanzen}

Bei der CITES ${ }^{17}$ (Konvention über den internationalen Handel mit gefährdeten Arten freilebender Tiere und Pflanzen) handelt es sich um ein internationales Handelsübereinkommen. Ziel ist es nicht, den Handel mit freilebenden Tieren oder Pflanzen zu verbieten, sondern so zu organisieren, dass ein Aussterben dieser Arten verhindert werden kann. Als Depositarstaat der Konvention übernimmt die Schweiz eine wichtige Rolle. Zudem befindet sich auch der Sitz des Sekretariats in Genf ${ }^{18}$. Das Übereinkommen ist seit Juli 1975 in Kraft und findet in 140 Staaten Anwendung. Harare, die Hauptstadt von Simbabwe, beherbergte vom 9. bis zum 20. Juni 1997 die zehnte Konferenz der CITES-Vertragsparteien. Die schweizerische Delegation wurde von Peter Dollinger, Chef der Abteilung Internationaler Verkehr des Bundesamtes für Veterinärwesen (BVET), geleitet.

Die Tagesordnung der Konferenz umfasste zahlreiche Verbesserungsvorschläge zu den Anhängen der Konvention. Die Konventionsbestimmungen sind auf rund 4000 Tierarten und über 30'000 Pflanzenarten anwendbar. Die Arten werden je nach geltenden Regelungen in drei Anhänge eingeteilt:

17. Convention on International Trade in Endangered species of Wild Fauna and Flora.

18. Secrétariat CITES, 15 chemin des Anémones, case postale 456, 1219 Châtelaine - Genf. Tel. 022/979 91 39, Fax $022 / 7973417$. 
- Anhang I: vom Aussterben bedrohte Arten, die durch den internationalen Handel beeinträchtigt werden könnten;

口 Anhang II : Arten, die vom Aussterben bedroht sein könnten, wenn der Handel nicht strikt reglementiert und kontrolliert wird;

- Anhang III: Arten, für welche besondere Regeln anwendbar sind, wenn sie von einer Partei angegeben werden.

Über 70 Arten wurden nach vorhergehender Überprüfung der Situation anders zugeordnet, neu in die Anhänge aufgenommen oder daraus gestrichen.

Bei der Evaluation der Arten trafen die Meinungen der Befürworter einer nachhaltigen Nutzung und der Verfechter eines absoluten Schutzes aufeinander. Die ersten sehen die sicherste Methode zur Gewährleistung des Überlebens der Tierarten darin, ihnen einen finanziellen Wert zuzuschreiben, so dass sie handelbar werden. Die zweiten dagegen betonen, dass die Regeln der Wirtschaft zwangsläufig eine missbräuchliche Nutzung und das Aussterben der Arten herbeiführen. Der Standpunkt der Schweiz besteht darin, den doppelten Grundsatz der Bewahrung und nachhaltigen Nutzung auszuwerten und dabei die von der CITES bereits festgelegten Kriterien zu berücksichtigen.

Die Öffentlichkeit interessierte sich vor allem für drei der rund siebzig evaluierten Arten: die afrikanischen Elefanten, die Wale und die weissen Nashörner. Der Status der beiden letzten Arten wurde auch nach den Verhandlungen nicht geändert. Anders verhält es sich für das Elfenbein, wo das 1990 verhängte Embargo auf Antrag dreier Länder (Simbabwe, Botswana und Namibia) aufgehoben wurde. Diese Länder erhielten die Bewilligung, nach einem Moratorium von 18 Monaten einen Teil ihrer Elfenbeinvorräte an Japan zu verkaufen. Die Aufhebung des Embargos wurde mit der Stimmenthaltung der Europäischen Union möglich. Die Schweiz hatte für den kontrollierten Handel mit Elfenbein abgestimmt.

Greenpeace wirft der Schweiz vor, die Konvention als Abkommen für einen freieren Handel und nicht als Artenschutzabkommen auszulegen. In einer sehr hitzigen Kampagne fordert Greenpeace den Rücktritt von Peter Dollinger als Repräsentant der Schweiz. Im August 1997 wurde dem Bundesrat eine Petition mit 15'000 Unterschriften vorgelegt. Der WWF Schweiz bedauert die Entscheidung von Harare, das Handelsembargo für Elfenbein aufzuheben, und bezeichnet sie als verfrüht. Der WWF distanziert sich jedoch deutlich von der Greenpeace-Kampagne.

\footnotetext{
QUELLEN

Übereinkommen über den internationalen Handel mit gefährdeten Arten freilebender Tiere und Pflanzen (CITES), Bundesamt für Veterinärwesen, Die Schweiz und die CITES, BVET, Juni 1997.

Eidgenössisches Volkswirtschaftsdepartement, Pressemitteilung, «Artenschutz-Konferenz (CITES) », 2. Juni 1997.

CITES, Press Note, August 1997.

Greenpeace, Pressemitteilung, «Dollinger weg - Artenschutz jetzt! », Bern, 14. Juli 1997.

La Liberté, 17. Juni 1997, «Une semaine décisive pour l'éléphant », 21. Juni 1997 «L'ivoire pourra à nouveau être l'objet de commerce».

Le Monde diplomatique, «Quelle rentabilité pour la faune sauvage?», Juni 1997.

Neue Zürcher Zeitung, 10. Juni 1997 «Streit um Elfenbeinhandel im südlichen Afrika», 15. Juli 1997 «Greenpeace auf Kopfjagd».
}

INTERNET-ADRESSEN

http://www. wcmc. org. uk/cites

http://www. unep. ch. 


\subsection{UMSETZUNG DER KONVENTION ÜBER KLIMAÄNDERUNGEN}

Das Endziel der Klimakonvention besteht laut Artikel 2 darin, «die Konzentration der Treibhausgase in der Atmosphäre auf einem Niveau zu stabilisieren, auf dem eine gefährliche anthropogene Störung des Klimasystems verhindert wird». Die COP-1 in Berlin beurteilte das Engagement der Industrieländer, dieses Ziel $z u$ erreichen, als ungenügend. Mit dem «Mandat von Berlin» beschloss die COP-1, für die Industrieländer mengenmässige Ziele zur Begrenzung und zur Reduktion der Treibhausgas-Emissionen nach einem genauen Zeitplan festzulegen. Die Ad-hoc-Arbeitsgruppe Berliner Mandat wurde damit beauftragt, ein Protokoll oder ein analoges rechtliches Instrument auszuarbeiten, welches das Engagement der Industrieländer präzisiert.

Bei der Konferenz über die Klimakonvention stand Entscheidendes auf dem Spiel. Der wissenschaftliche Bericht der zwischenstaatlichen Sachverständigengruppe über Klimaänderungen von Dezember $1995^{19}$ lässt daran keinen Zweifel. Die Erwärmung der Erdatmosphäre ist eine erwiesene Tatsache. Ihre Ursachen sind unter anderem die Verbrennung fossiler Energieträger (Erdöl und Kohle) sowie intensive Praktiken in der Land- und Forstwirtschaft. Anlässlich der 3. Konferenz der Vertragsparteien der Klimakonvention bemühte sich die internationale Gemeinschaft, den Trend der ansteigenden Emissionen umzukehren. Für die Konferenz von Kyoto wurden schwierige Verhandlungen erwartet, was denn auch der Fall war. Die Staaten konnten sich nur in extremis auf eine Einigung zum «Protokoll von Kyoto» verständigen.

Die verschiedenen Staaten oder Staatengruppen, die sich an der Konferenz beteiligten, traten mit deutlich unterschiedlichen Verhandlungspositionen an. Nur mit einem «Bravourstück» konnte ein Scheitern der Konferenz verhindert werden. Die Europäische Union forderte, dass die Industriestaaten die Emissionen der Treibhausgase, basierend auf dem Stand von 1990 um 7,5\% bis 2005 und um $15 \%$ bis 2010, reduzierten. Dazu sollen die Industrieländer verbindliche und harmonisierte Massnahmen verabschieden. Die Vereinigten Staaten, die weltweit grössten Emittenten von Treibhausgasen, kündigten in Japan an, den Ausstoss bis 2008 - 2012 zu stabilisieren und danach zu senken. Sie lehnten zwingende und mit andern Industrieländern harmonisierte Massnahmen ab und traten statt dessen für die Einführung ökonomischer Instrumente ein. Japan sowie die mittel- und osteuropäischen Länder verfolgten ehrgeizigere Reduktionsziele. Sie hatten Emissionssenkungen von 5\% bis 2008 - 2012 angekündigt. Der bekannte Nord-Süd-Gegensatz zeigte sich auch in Kyoto wieder: Die Entwicklungsländer und China betonten, dass das Treibhausgas-Problem von den Industrieländern verursacht worden sei und dass diese es demzufolge auch lösen müssten. Tatsächlich auferlegt das Berliner Mandat den Ländern des Südens keine neuen Verpflichtungen. Die Entwicklungsländer emittieren zwar gegenwärtig weniger Treibhausgase als die Industrieländer, aber im Jahr 2010 dürfte ihr Emissionsvolumen jenes der Industrieländer übersteigen.

19. IPPC, Second Assessment Report, Climate Change, 1995, Genf: OMM-UNEP, Dezember 1885. 
Ort und Zeitpunkt Hauptziele

Die Konferenz fand vom 1. bis zum 10. Dezember 1997 in Kyoto statt. Hauptziel der Konferenz war die Aushandlung eines Protokolls, das für die Industrieländer zwingende Reduktionsziele betreffend die Treibhausgas-Emissionen nach einem genauen Zeitplan vorschrieb (2005, 2010, 2020).

Weitere wichtige Punkte der Tagesordnung waren:

$\square$ Genehmigung eines internen Reglements der Vertragsparteien (COP):

$\square$ Änderung der Artikel 4.3 (Erweiterung der Finanzkompetenzen) und 17 (Festlegung des Mehrheitssystems für die Protokollgenehmigung);

$\square$ Änderung der Listen der Länder mit speziellen Verpflichtungen (Anhang I und II);

\ Verabschiedung des Budgets der Konvention;

$\checkmark$ Ausgestaltung der Finanzierungsmechanismen der Konvention mit dem Ziel, die Entwicklungsländer bei den Bemühungen zur Reduktion ihrer Treibhausgas-Emissionen zu unterstützen.

Teilnehmer

Die meisten der 167 Vertragsparteien der Konvention waren auf dem Gipfeltreffen von Kyoto vertreten. Vom 8. bis zum 10. Dezember fand der Ministerteil statt, währendem Regierungschefs und Minister die Verhandlungen abschlossen.

Schweizerische Delegation Die schweizerische Delegation an der COP-3 unter der Leitung von Beat Nobs, BUWAL, bestand aus Mitarbeitern des Bundesamtes für Umwelt, Wald und Landschaft, des Bundesamtes für Energie, des Departements für auswärtige Angelegenheiten und des Bundesamtes für Aussenwirtschaft. Auch verwaltungsexterne Kreise wurden eingeladen (Vorort, WWF Schweiz und Vertreter der akademischen Kreise). Bundesrätin Ruth Dreifuss und BUWAL-Direktor Philippe Roch vertraten die Schweiz während des Ministerteils.

Kontaktpersonen in der Bundesverwaltung José Romero, Abteilung Internationales, BUWAL, Tel. 031/322 6862 Markus Nauser, Einheit Klimaveränderungen, BUWAL, Tel. 031/324 4280

$\square$ Ergebnisse der Konferenz

Die zehntägigen Verhandlungen erwiesen sich als äusserst heikel. Unter grössten Schwierigkeiten verständigten sich die rund 160 Länder schliesslich auf eine Einigung in Form eines rechtlich verbindlichen Protokolls. Zum ersten Mal verpflichteten sich die Staaten auf zahlenmässige Reduktionsziele und auf einen Zeitplan. Das Protokoll enthält aber auch Verweise auf ökonomische Instrumente (handelbare Emissionszertifikate, Joint Implementation und Clean Development mechanism), die auf den starken Druck der Vereinigten Staaten aufgenommen wurden.

Die Industrieländer akzeptierten ausgehend vom Stand von 1990 eine Reduktion der Emissionen um durchschnittlich 5,2\% bis 2008 - 2012. Diese Reduktion als Ganzes wird differenziert auf die einzelnen Länder verteilt: $8 \%$ für die Schweiz, die Europäische Union und die meisten mittel- und osteuropäischen Länder; $7 \%$ für die Vereinigten Staaten, $6 \%$ für Kanada, Ungarn und Japan. Bestimmte Länder werden jedoch sogar berechtigt, ihre Emissionen zu erhöhen (Australien um $8 \%$, Norwegen um $1 \%$ ). 
Das Protokoll bewilligt neue ökonomische Instrumente. Mit «handelbaren Emissionszertifikaten», allgemein als «Verschmutzungsrechte» bekannt, können Länder, die ihren Plafond überschreiten, Emissionsrechte von Ländern abkaufen, die ihr Kontingent nicht ausgeschöpft haben. Unter «Joint Implementation» (gemeinsame Umsetzung) wird eine Form der Zusammenarbeit zwischen Ländern verstanden, die einem Land erlaubt, einen Teil seiner Emissionsreduktion durch private oder öffentliche Investitionen in Projekte in einem anderen Land zu realisieren. Von der Joint Implementation wird eine Verbesserung des KostenNutzen-Verhältnisses klimarelevanter Investitionen sowie ein verstärktes Engagement des privaten Sektors erwartet. Der «Clean Development Mechanism» soll die gemeinsame Umsetzung der Industrie- und der Entwicklungsländer ermöglichen. In Kyoto wurde zwar das Prinzip einer Einführung solcher Instrumente gutgeheissen, aber es wurden keine Funktionsmechanismen für diesen neuen Markt definiert oder verabschiedet. Das soll anlässlich der nächsten Klimakonferenz im November 1998 in Buenos Aires geschehen. Bis dahin kann also kein Land mit solchen Emissionsrechten handeln.

Das Protokoll in seiner definitiven Form kommt sowohl der Europäischen Union entgegen, die in Zahlen ausgedrückte Reduktionsziele wünschte, als auch den Vereinigten Staaten, welche die Anerkennung ökonomischer Instrumente forderten. Diese handelbaren Zertifikate würden ihnen ermöglichen, "Verschmutzungsrechte» von Ländern zu kaufen, die weniger Treibhausgase emittieren als bewilligt. Das Protokoll, auf das man sich einigte, liegt nun den Vertragsparteien zur Unterzeichnung auf. Die Vereinigten Staaten haben ihre Unterzeichnung bereits von der Beteiligung der wichtigsten Entwicklungsländer am Prozess der Emissionssenkung abhängig gemacht.

\section{$\square$ Haltung der Schweiz}

Die Schweiz nimmt das Problem der Klimaänderungen sehr ernst. Aufgrund der bereits in der Klimapolitik angewandten Massnahmen vertrat sie die glaubwürdigste Position, um Reduktionsziele für $\mathrm{CO}_{2}$ auszuhandeln. Die Schweiz beabsichtigt, ihre $\mathrm{CO}_{2}$-Emissionen dank einer Palette von Massnahmen bis zum Jahr 2000 um $10 \% \mathrm{zu}$ reduzieren (ausgehend vom Stand von 1990). Die Umwelt-, Energie(Energie 2000), Verkehrs- und Finanzpolitik enthält jeweils ein Kapitel zum Klimaschutz. Das ehrgeizigste Projekt ist zweifellos der Entwurf eines $\mathrm{CO}_{2}$-Gesetzes.

Eine Untersuchung der Auswirkungen der schweizerischen Klimapolitik für die Jahre 1990 bis $1995^{20}$ zeigt, dass die $\mathrm{CO}_{2}$-Emissionen stabil geblieben sind und dass die Schweiz im Jahr 2000 eine Stabilisierung auf dem Stand von 1990 erzielen dürfte.

Gestützt auf diese Leistungen setzte sich die Schweiz anlässlich der COP-3 für eine ehrgeizige Position ein ${ }^{21}$. Auf einer Pressekonferenz wurden verschiedene Vorschläge vorgestellt, welche die Schweiz befürwortet und in das Klimaprotokoll aufnehmen will:

20. Bundesamt für Umwelt, Wald und Landschaft, UN Frame Convention on Climate Change - Second national communication of Switzerland 1997 - Greenhouse Inventory 1995, Swiss Confederation, Bern, April 1997.

21. BUWAL, Pressemitteilung, «Schweiz will verbindliches Klimaprotokoll», Bern, 11. November 1997. 
- Vorsorgeprinzip. Trotz noch offener wissenschaftlicher Fragen sollen Strategien und Massnahmen zur Reduktion der Treibhausgas-Emissionen eingeleitet werden.

๑ Verursacherprinzip. Die Länder mit den höchsten Pro-Kopf- Emissionen sollten die grössten Anstrengungen unternehmen.

๑ Zudem sollte das Protokoll die folgenden Grundsätze verankern:

口 eine signifikante Gesamtreduktion der drei Treibhausgase Kohlendioxid, Methan und Lachgas anstreben;

๑ entsprechend dem Verursacherprinzip nach Ländern differenzierte Verpflichtungen festlegen;

๑ für die Länder gemäss Anhang I der Konvention (Industrieländer sowie Staaten Zentral- und Osteuropas) verpflichtende, harmonisierte und international koordinierte Strategien und Massnahmen vorschreiben, um eine Gesamtreduktion der Emissionen um 10\% zu erzielen.

\section{ENTWURF EINES $\mathrm{CO}_{2}$-GESETZES}

Ein neues Bundesgesetz über die Reduktion der $\mathrm{CO}_{2}$-Emissionen wird derzeit im Parlament erörtert E(Sessionen 1997, Frühling 1998). Aufgrund dieses Gesetzesentwurfs - ein wichtiges Element der schweizerischen Klimapolitik - soll bis im Jahr 2010 der $\mathrm{CO}_{2}$-Ausstoss um 10\% unter den Stand von 1990 gesenkt werden. Während für Treibstoffe eine Reduktion von 5\% angestrebt wird, soll jene für Brennstoffe $15 \%$ betragen.

Zur Erreichung dieser Ziele werden bereits beschlossene oder geplante Massnahmen zur Reduktion der $\mathrm{CO}_{2}$-Emissionen in den Sektoren Energie, Verkehr und Landwirtschaft berücksichtigt. Das Gesetz soll auch freiwillige Massnahmen und Verpflichtungen der Wirtschaftskreise fördern. Für 2004 ist eine Evaluation geplant. Falls die Ziele mit diesen Mitteln nicht erreicht werden, sieht der Gesetzesentwurf die Einführung einer $\mathrm{CO}_{2}$-Abgabe vor.

Die Schweiz ist derzeit das einzige Land, wo das Parlament sich mit einem Gesetzesentwurf über $\mathrm{CO}_{2}$ befasst.

QUELLE

"Botschaft zum Bundesgesetz über die Reduktion der C02-Emissionen vom 17. März 1997 ", Botschaft Nr. 97.030.

Ferner setzt sich die Schweiz für die Aufnahme der Joint Implementation in das Klimaprotokoll ein.

Insgesamt ist die Schweiz mit den Ergebnissen von Kyoto und hauptsächlich mit den von den Industrieländern akzeptierten Reduktionszielen zufrieden. Für die Schweiz entspricht das im Protokoll definierte Ziel dem Ziel des Gesetzesentwurfs. Die Ratifizierung ist daher im Prinzip bereits gesichert.

Die an der Konferenz von Kyoto anwesenden Umweltorganisationen äusserten ihre Enttäuschung über die von den Regierungen beschlossene Einigung. Der WWF Schweiz spricht sogar von «zu grossen Schlupflöchern » ${ }^{22}$ und beschreibt das Abkommen als wenig überzeugendes Papier voller Klauseln, die den «verschmutzenden» Staaten erlauben, weiter Treibhausgase in die Atmosphäre auszustossen. Der WWF Schweiz bedauert, dass der in Kyoto paraphierte Vertrag die Gefahr einer Klimaänderung nicht abwendet, weil die Beteiligten - vor allem Japan und die Vereinigten Staaten - eine Definition realistischer Ziele zur Reduktion der umweltbelastenden Emissionen ablehnten. 
@ JSDW 1993, S. 67 - 69 (Beschreibung der Konvention).

U. JSDW, 1994, S. 66 - 67 (Erste Ratifizierung der Konvention, Beschreibung von Energie 2000)

凹SDW 1995, S. 68 - 71 (Ratifizierung der Konvention durch die Schweiz, Energie 2000/CO 2 -Abgabe)

凹SDW 1996, S. 52 - 58 (Konferenz von Berlin, Übersicht, $\mathrm{CO}_{2}$-Abgabe)

[D JSDW 1997, S. 60 - 64 (Bericht des IPCC, Zweite Vertragsparteienkonferenz)

\section{QUELLEN}

Bundesamt für Umwelt, Wald und Landschaft, UN Frame Convention on Climate Change - Second national communication of Switzerland 1997 - Greenhouse Inventory 1995, Swiss Confederation, Bern, April 1997.

Bundesamt für Umwelt, Wald und Landschaft, Klima in Gefahr - Fakten und Perspektiven zum Treibhauseffekt,

BUWAL, September 1997.

Bundesamt für Umwelt, Wald und Landschaft, Pressemitteilung, «Schweiz will verbindliches Klimaprotokoll», Bern, 11. November 1997.

United Nations Environment Programme, Climate Change Information Kit, UNEP, Januar 1997.

Climate Change Secretariat, Press Kit - Kyoto 1997, Oktober 1997.

Fritz Gassmann, Was ist los mit dem Treibhaus Erde, Ed. vdf Zürich und Treubner Stuttgart, Leipzig, 1994.

\section{INTERNET-ADRESSEN}

Server der Konvention über Klimaänderungen: http://www. unfccc. de

Server des UNEP für internationale Umweltschutzkonventionen: http://www. unep. ch/iuc. html.

Server des Bundesamtes für Umwelt, Wald und Landschaft: http://www. admin. ch/buwal 